\title{
Der Einfluß roter und blauer Strahlung auf die Ausbildung der Chloroplasten bei gehemmter Proteinsynthese
}

\author{
Von Rainer Bergfeld \\ Aus dem Botanischen Institut der Universität Freiburg i. Br. \\ (Z. Naturforschg. 19 b, 1076-1078 [1964]; eingegangen am 27. August 1964)
}

\begin{abstract}
In earlier papers ${ }^{1,2}$ it has been shown that morphogenetically effective blue light - compared with red light - increases protein synthesis in young fern gametophytes (= sporelings). In the present paper evidence has been presented that much of this protein which is synthesized under the influence of blue light is located in the chloroplasts. The size of the chloroplasts is correlated with the protein contents of the sporelings. Growth of the chloroplasts can be blocked by antimetabolites like 5-methyl-tryptophane, which inhibit protein synthesis. The block can be reversed by the application of tryptophane in a suitable concentration. It can be concluded from the data of this paper that only part of the protein, which originates under the influence of blue light can be regarded as specific enzyme protein in the cytoplasm. Much of the blue light dependent protein rather seems to be structural protein of the chloroplasts.
\end{abstract}

Bei der Entwicklung der Vorkeime (= Gametophyten) des Farnes Dryopteris filix-mas beobachtet man eine enge Korrelation zwischen Proteinsynthese und Morphogenese ${ }^{1,2}$. Durch kurzwellige sichtbare Strahlung (= Blaulicht) kann bei diesen Pflanzen die Proteinsynthese gesteigert werden. Gleichzeitig kommt es zur Ausbildung „normaler“ zwei- bis dreidimensionaler Prothallien. Im längerwelligen Licht (Hellrotlicht) wachsen die Vorkeime zu Zellfäden aus.

Im Zusammenhang mit der durch Blaulicht induzierten normalen Morphogenese beobachtet man auch eine Vergrößerung der Chloroplasten ${ }^{3}$. Es ist anzunehmen, daß die Chloroplasten-Vergrößerung in den mit Blaulicht bestrahlten Vorkeimen mit einer vermehrten Proteinbildung in den Chloroplasten zusammenhängt ${ }^{4}$. In den vorliegenden Versuchen wurde den Vorkeimen ein Inhibitor der normalen Proteinsynthese zugesetzt, - Methyltryptophan - , um Zusammenhänge zwischen Proteinsynthese und Chloroplastenausbildung zu finden. Durch Zugabe des normalen Metaboliten Tryptophan konnten die Effekte des Antimetaboliten wieder revertiert werden.

\section{Material und Methode}

Das verwendete Sporenmaterial, die Keimungsinduktion sowie die Kulturbedingungen waren die gleichen

1 K. Ohlenroth u. H. Mohr, Planta 59, 427 [1963].

2 K. Ohlenroth u. H. Mohr, Planta 62, 160 [1964].

3 R. Bergfeld, Z. Naturforschg. 18 b, 328 [1963]. wie in einer vorangegangenen Arbeit ${ }^{4}$. Als Antimetabolit der Proteinsynthese wurde DL-5-Methyltryptophan (Fluka AG. Buchs-Schweiz) verwendet. Die Applikation erfolgte bei Versuchsbeginn (= Einsetzen in die Lichtfelder). Bei Revertierungs-Versuchen wurde das DLTryptophan sieben Tage nach Versuchsbeginn zugegeben. Das DL-5-Methyltryptophan gelangte in drei Konzentrationen zur Anwendung, und zwar so, daß die Endkonzentrationen in den Kulturmedien $2 \cdot 10^{-4}$, $1 \cdot 10^{-4}$ und $2,5 \cdot 10^{-5}-m$. Lösungen entsprachen. Bei Revertierungs-Versuchen wurde das DL-Tryptophan anfangs in aequimolaren Konzentrationen appliziert, bei weiteren Versuchen wurden die Konzentrationen, im Vergleich zum DL-5-Methyltryptophan, erhöht. Näheres ist bei den einzelnen Versuchen angegeben. Die Messungen der Chloroplasten in der Basalzelle der Vorkeime erfolgten am 7., 14., 21. und 28. Tag nach Versuchsbeginn mittels Okularmikrometer bei einer geeigneten Vergrößerung $(10 \cdot 2 \cdot 100)$. Die Meßpunkte bei den graphischen Darstellungen wurden aus den Mittelwerten von sechs Meßreihen (= Parallelversuchen) zu je 50 Messungen errechnet. Der mittlere Fehler wurde wie üblich berechnet. Die Differenz zweier Werte wurde als signifikant betrachtet, wenn $P \leqq 0,05$ war.

\section{Experimentelle Ergebnisse}

\section{A. Die Entwicklung der Chloroplasten bei Ein- wirkung von Methyltryptophan (M-Try)*}

Die Versuche zeigen, daß im HR die Chloroplasten-Durchmesser durch die Einwirkung von M-Try geringfügig abnehmen. Die Differenzen zwi-

${ }^{4}$ G. Brawermann, A. O. Pogo u. E. Chargaff, Biochim. biophysica Acta [Amsterdam] 48, 418 [1961].

* Folgende Abkürzungen werden verwendet: HR $=$ Hellrot licht, $\mathrm{Bl}=$ Blaulicht, M-Try $=\mathrm{DL}-5-$ Methyltryptophan, Try $=$ DL-Tryptophan. 
schen den entsprechenden Werten sind signifikant, z. B. bei 14-tägiger Kultur mit $P=0,015$ (Abb. 1). Die Ergebnisse deuten darauf hin, daß unter normalen HR-Bedingungen eine geringe Proteinsynthese in den Chloroplasten erfolgt, die durch M-Try gehemmt werden kann. Die mit den drei angewandten Konzentrationen von M-Try erzielten Effekte waren nicht signifikant verschieden.

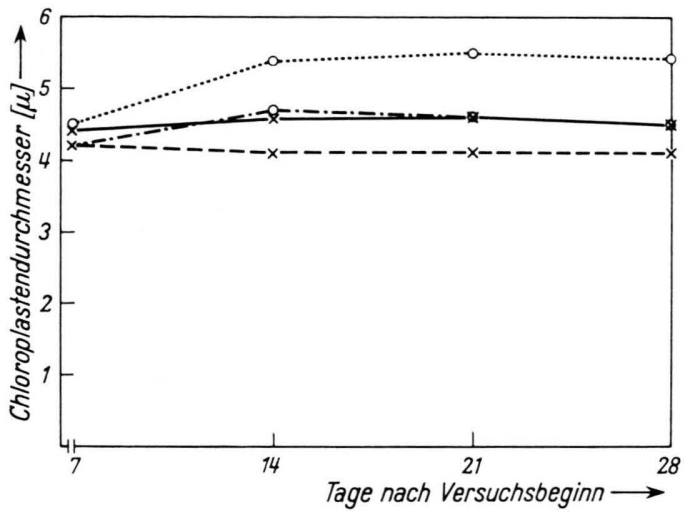

Abb. 1. Die Änderung der Chloroplasten-Durchmesser in der Basalzelle von Hellrot-Vorkeimen bei Einwirkung von $2,5 \cdot 10^{-5}-m$. M-Try $(X--X) ; 2 \cdot 10^{-4}-m$. Try $(0 \cdots \cdots)$; $2,5 \cdot 10^{-5}-m$. M-Try $(7$ Tage $)+2 \cdot 10^{-4}-m$. Try $\left(\mathrm{O}_{-} \cdot-\cdot-\mathrm{O}^{\circ}\right)$; unbehandelte Kontrolle $(\times-\times)$.

Wesentlich augenfälliger sind die Ergebnisse bei den entsprechenden Bl-Versuchen. Die Chloroplasten der Bl-Zellen reduzieren ihren Durchmesser unter der Einwirkung von M-Try nahezu auf die Größe der unbehandelten HR-Chloroplasten. Die Differenz bei 14-tägiger Kultur ist gegenüber Bl-Kontrollen

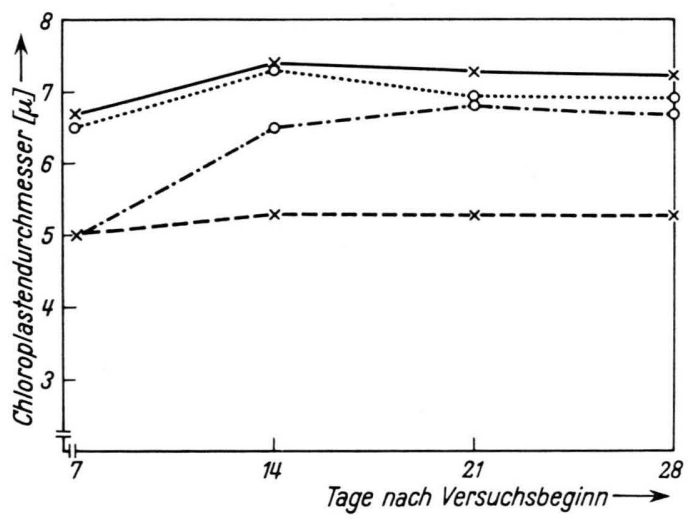

Abb. 2. Die Änderung der Chloroplasten-Durchmesser in der Basalzelle von Blaulicht-Vorkeimen bei Einwirkung von $2,5 \cdot 10^{-5}$-m. M-Try $(\times--\times) ; 2 \cdot 10^{-4}-m$. Try $(0 \cdots \cdots)$; $2,5 \cdot 10^{-5}-m$. M-Try $(7$ Tage $)+2 \cdot 10^{-4}-m$. Try $\left(O_{--} \cdot \cdot-O\right)$; unbehandelte Kontrolle $(\times-X)$. mit $P \leqq 0,0005$ gesichert, gegenüber den HR-Kontrollen mit $P=0,04$ eben noch signifikant (Abb. 2). Wichtig ist, daß augenscheinlich die Chloroplastenzahl sowohl in den mit M-Try behandelten HR- als auch in den Bl-Zellen nicht merklich beeinflußt wird. Die Chloroplasten sind auch bei Zugabe des Antimetaboliten in den Bl-Zellen zahlreicher und dichter gelagert als in den HR-Zellen.

\section{B. Das Verhalten der durch Methyltryptophan (M-Try) gehemmten Chloroplasten bei Einwirkung von Tryptophan (Try)}

Im Gegensatz zur Teilungsaktivität der Zellen, die ebenfalls durch M-Try herabgesetzt wird (BERGFELD, in Vorbereitung), reagieren die Chloroplasten relativ schnell auf den Zusatz von Try. Ein unterschiedliches Verhalten zeigen aber die gehemmten HRund Bl-Chloroplasten hinsichtlich des Ausmaßes der Revertierung. Die HR-Chloroplasten, selbst wenn sie durch die stärkste M-Try-Konzentration gehemmt wurden, vergrößern sich bei Zusatz von aequimolaren Try-Lösungen auf den Durchmesser unbehandelter Chloroplasten; dies gilt auch für die Versuche mit $2,5 \cdot 10^{-5}-m$. M-Try und nachfolgender Behandlung mit $2 \cdot 10^{-4}-m$. Try (Abb. 1). Bei all diesen Versuchen war sieben Tage nach Try-Applikation kein signifikanter Unterschied gegenüber den unbehandelten Chloroplasten festzustellen.

Im Bl erfolgt bei Zusatz einer aequimolaren Lösung Try eine Vergrößerung der Plastidendurchmesser, es wird jedoch nicht ganz der Kontrollwert erreicht. Setzt man nach vorheriger M-Try-Behandlung mit $1 \cdot 10^{-4}-m$. oder $2 \cdot 10^{-4}-m$. die doppelte Konzentration von Try zu, so vergrößern sie sich nahezu bis zur Größe der entsprechenden unbehandelten Kontrolle. Die Differenz ist nach 21 Tagen Try-Einwirkung mit $P=0,02$ jedoch noch gesichert. Bei den Versuchen mit $2,5 \cdot 10^{-5}$-m. M-Try und nachfolgender Applikation von $2 \cdot 10^{-4}-m$. Try wird im $\mathrm{Bl}$ nach 21 Tagen Try-Einwirkung der Kontrollwert nahezu erreicht (Abb. 2).

Bemerkenswert ist, daß bei alleiniger Zugabe von Try die Chloroplasten im HR, nicht aber im Bl, gröBere Durchmesser erreichen als bei den Kontrollen. Die Größenzunahme ist schon nach 14 Tagen mit $P \leqq 0,0005$ signifikant. Dieser Befund deutet darauf hin, daß im HR, nicht aber im Bl, das Try als ein begrenzender Faktor für die Intensität der Proteinsynthese in den Chloroplasten anzusehen ist. 


\section{Diskussion}

Die Resultate dieser Arbeit zeigen, daß die GröBenveränderungen der Chloroplasten in den Farnvorkeimen als ein unmittelbarer Ausdruck unterschiedlicher Proteinsynthese angesehen werden können. Ein wesentlicher Teil des im Blaulicht vermehrt gebildeten Proteins findet sich in den Chloroplasten. Es ist nicht wahrscheinlich, daß durch das morphogenetisch wirksame Blaulicht lediglich die Synthese von Enzymproteinen veranlaßt wird. Das in den vorliegenden Untersuchungen gefundene Verhalten der Chloroplasten deutet vielmehr darauf hin, daß ein beträchtlicher Teil des im $\mathrm{Bl}$ gebildeten Proteins als Strukturprotein der Chloroplasten aufzufassen ist. - Auch beim Wachstum der Vorkeime im HR findet in den Chloroplasten eine gewisse Proteinsynthese statt, die durch M-Try gestört, durch Zugabe von Try aber rasch wieder auf den normalen Wert gebracht werden kann. Im Bl können die durch M-Try verursachten Störungen durch Try nicht völlig revertiert werden. Die verbleibende Differenz ist allerdings nicht befriedigend signifikant. Offenbar verursacht M-Try einige irreversible Defekte in den Chloroplasten.

Zusammenfassend kann man sagen, daß ein beträchtlicher Teil der unter dem Einfluß von $\mathrm{Bl}$ in den Zellen der Farnvorkeime entstehenden Proteine in den Chloroplasten gelagert ist.

Die Arbeit wurde mit Hilfe der D e u t s c h e n F or $\mathrm{schung}$ g e meinschaft (Leihgaben an Herrn Prof. Монг) durchgeführt. 